\title{
Overview of Technical Elements of Liver Segmentation
}

\author{
Nazish Khan* \\ Institute of Management Sciences, \\ Peshawar, Pakistan \\ Imran Ahmed \\ Institute of Management Sciences, \\ Peshawar, Pakistan
}

\author{
Mehreen Kiran \\ Institute of Management Sciences, \\ Peshawar, Pakistan \\ Awais Adnan \\ Institute of Management Sciences, \\ Peshawar, Pakistan
}

\begin{abstract}
Liver diseases are life-threatening, it's important to detect it tumor in early stages. So, for tumor detection Segmentation of the liver is a first and significant stride. Segmentation of the liver is a yet difficult undertaking in view of its intra patient variability in intensity, shape and size of the liver. The aim of this paper is to assemble a wide assortment of techniques and used CT scan dataset information for liver segmentation that will provide a decent beginning to the new researcher. There are different strategies from basic to advance like thresholding, active contour, region growing to graph cut is briefly abridge to give an outline of existing segmentation strategies. We review the concept of particular strategies and review their original ideas. Our idea is to provide information under which condition a chosen strategy will work or utilize.
\end{abstract}

Keywords-component; CT Scan; Liver; Dataset; Segmentation technique

\section{INTRODUCTION}

The liver is key and biggest organ among different organs of the body and provide exceptionally crucial task to our body to keep it free from toxins and harmful substances such as alcohol and medications. Its primary vital functions are: to filter the blood coming from digestive track, it supports all other organs of the body in some way, regulate the supply of body fuel by managing glucose level in our body, cleanse the blood by metabolizing alcohol and obliterating and neutralizing destructive substances, produce bile, which helps in digestion, manufacture many primary and essential proteins which provide resistance to infection and help in blood clotting and thickening. Its direct the supply of vitamins and mineral in our body.

According to Global Cancer Statistics [26] liver cancer fifth the most commonly diagnosed and the second driving reason for death among men and seventh in women. Distinct methods for liver cancer is a blood test, screening and biopsy. A biopsy is intrusive procedure and is exceptionally painful diagnostic technique for patients. That is why researchers are attempting to develop noninvasive techniques. In this way, before the detection of the liver pathology, liver must be segmented accurately.
Liver segmentation is challenging task to develop robust strategies for liver segmentation. Researchers are coping with this challenge to automatically segment the liver; exceedingly unique shapes and volume of liver, similar intensity value among adjacent organs (stomach, spleen, Aorta and abdominal wall), complicated liver structure and contrast media injection cause liver tissue to have different grey level value. Liver segmentation is still an open problem as a result of these challenges. At the time being, researchers are dealing with challenging tasks to increase accuracy in diagnosis and maintain the strategic distance from the need for biopsy (a small tissue of tumor is removed and analyzed) and surgery. These systems do not replace the radiologist, but only provide the second opinion in diagnosis and support radiologist to settle on their choice. Segmentation methods are categorized into 2 main categories; automatic and interactive method. An automatic method has no user intervention and are fully automated and free from user error. It potentially saves the time of operators. The semi-automatic required user intervention like refinement of binary mask and in the selection of seed points. General approach to deal with follow in automated and semi-automatic CAD (Computer Aided System) system is: Pre-processing, Liver segmentation, Lesion Detection, Feature extraction, Classification, Evaluation. Some paper applies the post processing also as indicated by their prerequisite. In pre-processing phase filters are utilized to enhance the image quality and features, furthermore to remove noise and contortion from images. liver segmentation, the liver is sectioned from other encompassing organs. Lesion are detected from segmented liver. Then features are extracted from the lesion area to categorize them either benign or malignant, primary or secondary tumors.

Different modalities are utilized for the diagnosis of liver pathology (liver cancer, cirrhosis, hepatitis) such as CT (Computed Tomography), MRI (Magnetic Resonance Imaging), (US)ultrasounds. According to research CT is the most preferred modality because CT is less costly than MRI. The CT data is collected in the form of DICOM (Digital Imaging and Communication in Medicine) image. DICOM images can be converted to many types, however, for medical 
imaging it is ideal to utilize .PNG or .BMP. Many researchers work on Liver segmentation and propose different techniques. Each technique has its own merits and shortcomings.

The rest of the Paper is organized as follows section II Literature Review, section III we organize liver segmentation techniques overview, section IV we include conclusion to summarize our views. we place the dataset comparison table and techniques overview table at the end of paper.

\section{LITERATURE REVIEW}

Lim et al [2] Develop automatic liver segmentation using previous knowledge of liver position and use a deformable contour method based on morphological filtering operation. On the gradient label map Algorithm [2] perform deformable contouring. To reduce computational complexity and to decide suitable threshold histogram analysis is performed in the ELP (Estimated liver position). Proposed method uses multi scale morphological filter recursively with region labeling and clustering to detect search rang for deformable contouring. They use private dataset of 10 patients. Results are compared with manual segmentation by a radiologist. Graphical representation is shown below in Fig. 1

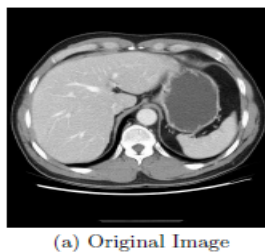

(a) Original Image

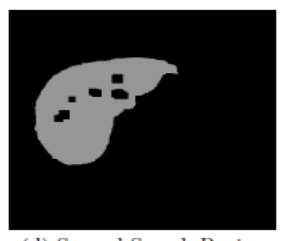

(d) Second Search Region
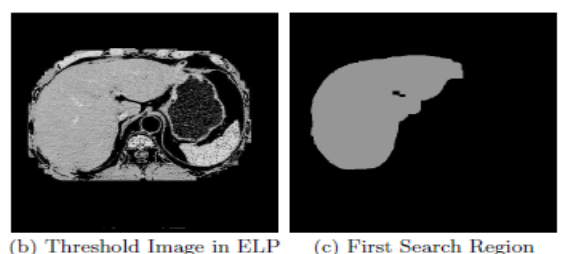

b) Threshold Image in ELP

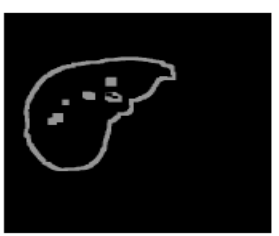

(e) Search Range
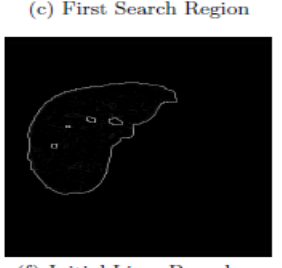

(f) Initial Liver Boundary

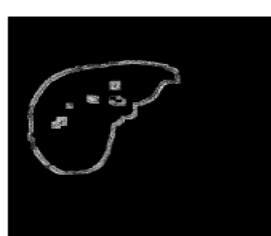

(g) Gradient-label Map

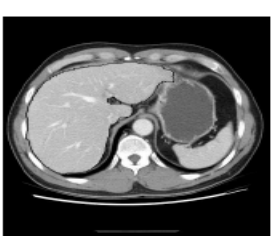

(h) Result Image
Fig. 1. Final Segmentation result

Suzuki et al [4] developed automatic liver extraction technique for contrast enhance CT images. The anisotropic diffusion filter uses for image denoising and preserving the shape of the liver. Scale specific gradient magnitude use to enhance liver boundary. These preprocessing results are passed to fast marching level set algorithm that initially refines the liver boundary and use as a rough estimate of liver shape and geodesic active contour combine with level set to extract liver shape. And estimate liver volume. Liver manually trace by the expert radiologist is use as a gold standard to compare the evaluation results of liver volume. Their local dataset consists of 15 patients. Overall accuracy is $98.4 \%$. Sensitivity, specificity and percent volume error is $91.1 \%$, $99.1 \%$, and 7.2 respectively. Limitation of the paper is small dataset. Graphical representation is shown in Fig. 2

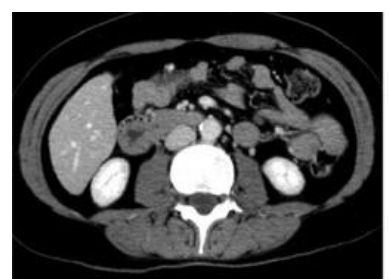

(a)

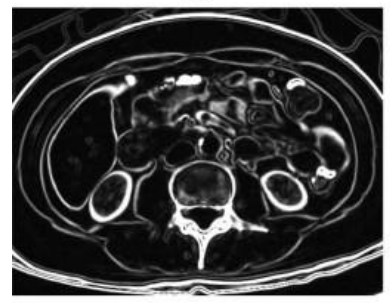

(c)

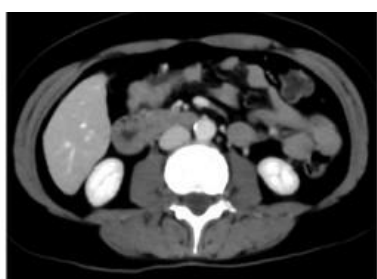

(b)

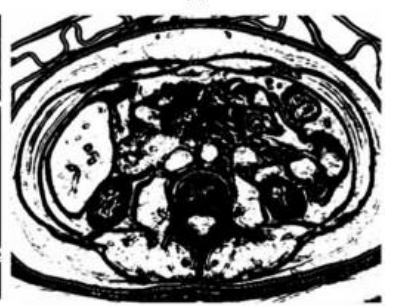

(d)

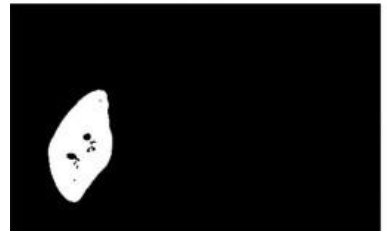

Fig. 2. (a) Original CT image (b)Anisotropic diffusion noise reduction (c) Scale specific gradient magnitude calculation (d) Non-linear grey scale conversion(e) Geodesic active contour segmentation

Massoptier et al [7] Develop innovative statistical model based approach. Active contour and gradient vector flow (GVF)are also used for hepatic segmentation. They analyzed nonlinear anisotropic diffusion and mean shift filter based on the processing time. To save processing time they follow mean shift filter. Clustering technique was used with their powerful initialization method for hepatic lesion segmentation. For lesion detection $82.6 \%$ Sensitivity and $87.6 \%$ specificity were achieved. Liver volume overlapping was evaluated by DSC (Dice Similarity Coefficient), FNR (false negative ratio), FPR (false positive ratio). They use their own private data set of 21 patient CT data set. From 46 lesions, they diagnosed different type of tumor 6 were HCC (Hepatocellular carcinoma), 2HDG (Hemangioma), 8HM (Hepatic metastases) and 5 have healthy patients.

Yussof et al [10] proposed automatic 3D liver segmentation algorithm using a hybrid technique that combines morphological operations with graph cut method. Anisotropic diffusion filter for noise removal.In liver region estimation histogram analysis was performed and give their assumption that liver, grey value always lies between 75 to 200.For liver segmentation 2D and 3D Connected Component Labeling was performed. The graph cut technique was used for the refinement of CCL segmented liver and for reconstruction of liver surface. Accuracy check is performed in 10 cases of silver07 dataset. Computation time is less than 6 min. For evaluation of segmentation results, use 5 different evaluation metrics. Volumetric overlap error(VOE), Relative volume difference(RVD), Average symmetric surface difference(SD), Maximum symmetric surface difference (MSD) and Root mean square symmetric surface difference(RMSD). On average results VOE is less than $10 \%$, RVD is $2.99 \%$ and RMSD is close to $2 \mathrm{~mm}$.Limitation of their 
paper is, they face under segmentation problem when lesions are close to liver boundary. The dataset is very small.

Militzer et al [12] Proposed a novel system for automatically detecting and segmenting focal liver region from CT images. For classification, it utilizes a probabilistic boosting and thus provide fully automated detection and segmentation of the liver lesion simultaneously. They use hierarchical mesh-based shape representation for liver segmentation. Features selected in this paper are gray level statistical feature and Haar like features. Detection rate $77 \%$ could be achieved with the specificity of $0.93 \%$ and a sensitivity of $0.95 \%$ at the same time for lesion segmentation at the same setting.

S.S Kumar [15] presented an automatic segmentation of the liver lesion from CT radiographs. This paper utilizes medium filter, erosion, dilation, largest connected component as a pre-processing step. In post-processing, morphological operators are utilized to additionally refine the image and utilize basic region growing techniques for live segmentation and an alternate Fuzzy C-Means Clustering for tumor segmentation. He used 10 cases in his research work. The Technique result was contrasted and evaluated with the manual segmentation based on false positive rate, false negative rate, volume measurement error, spatial overlap and visual overlap. Pictorial results are shown in Figure .1 below.

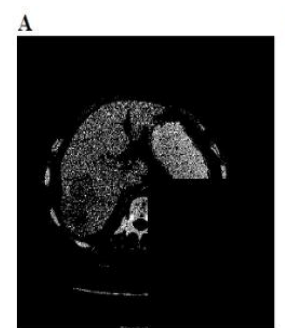

Simplified Image

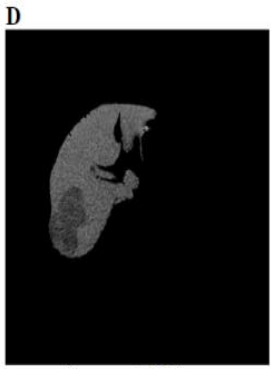

Segmented Liver

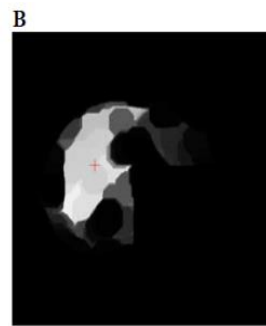

Eroded Image with Centroid

(a)

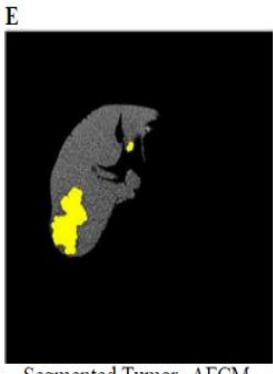

Segmented Tumor -AFCM

(b)

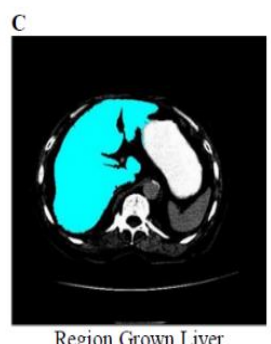

Region Grown Liver

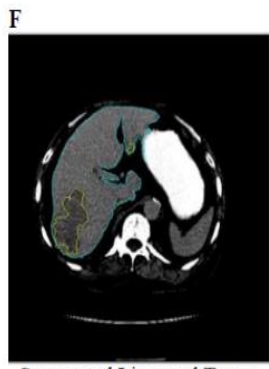

Segmented Liver and Tumor liver segmentation using AFCM

Belgherbi et al [17] A semi-automatic method developed for liver lesion extraction using mathematical morphology from CT images. In pre-processing for refinement of the liver they use dilation, erosion and anisotropic diffusion filter. For liver lesion, they use mathematical morphology, especially on the water shed technique. They use private data set. The proposed scheme achieves $92 \%$ Sensitivity \& 99\% Specificity. Brief graphical representation is shown in Fig.4. Here we focus only on the graphical representation of the liver. Pictorial representation of liver lesion is not represented here.

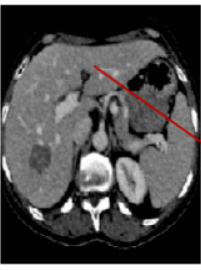

a

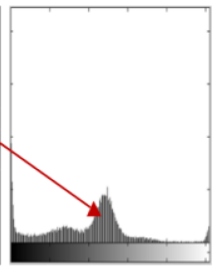

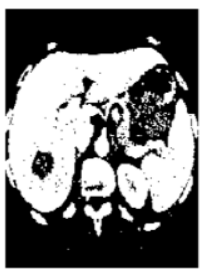

$c$

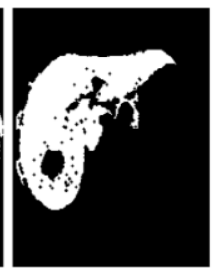

d
Fig. 4. Original image Histogram Thresholding Liver extraction

Marcin Ciecholewski [18] present novel method which automatically segments the liver shape. In CT scan images lumber section of the spine is utilized as seed point. After seed point selection, joint polylines are drawn to approximate the liver contour. These component polylines frames the components of two polygons eliminated from the image, which leaves only the segmented liver shape inside the image. Results are accessed on 13,30 images using Dice's similarity. Fig. 5 shows the results graphically.

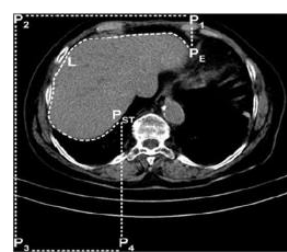

(a)

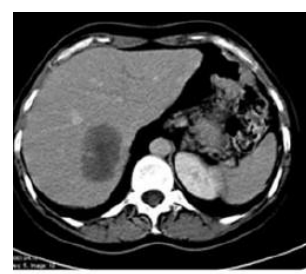

g

CT images

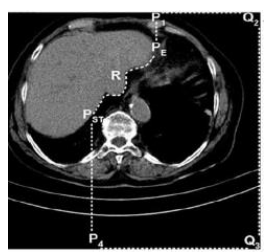

(b)

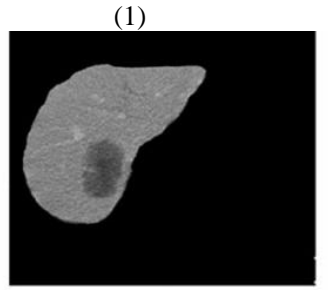

h

Ground truth

(2)

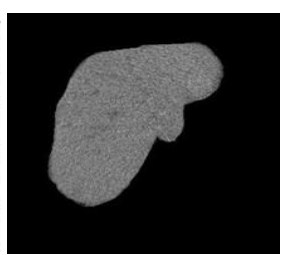

(c)

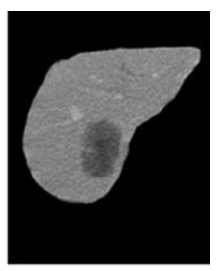

proposed method

Fig. 5. (1) (a)Polygon with polyline L(b) Polygon with polyline $\mathrm{R}(\mathrm{c})$ segmented liver (2) Segmentation of liver shape using connected polylines

Anter et al [20] proposed hybrid approach using adaptive threshold and CCL for liver segmentation. For liver lesion segmentation, their method based on watershed [14] and region growing. RG algorithm has their limitation such as initial point position and its selection highly affects the segmentation result if they are not properly handling well. So, to overcome these limitations the integrate RG with watershed algorithm. Their 2 dataset consist of 112 Patients, one of radiopaedia website and aother is a local dataset collected from a local hospital. The computational time is $0.15 \mathrm{~s} / \mathrm{slice}$. Overall, liver extraction accuracy is 93\%. (CY) cyst (hepatocellular carcinoma), (HG)hemangioma, (HA) hepatic adenoma, (FNH) Focal nodular hyper plasma, (CC) cholangiocarcinoma, (MS)metastases achieve the accuracy $0.91,0.90,0.93,0.95,0.91,0.94,0.94 \%$ respectively. 
Aldeek et al [21] develop semiautomatic method using a Bayesian classifier for liver segmentation. In post processing, median filter and some filling operation are performed to further refine the classifier results. Dataset consists of 44 cases. Average area overlaps accuracy is over $87 \%$ They evaluate their results with the manual segmentation done by the expert radiologist. Graphical representation is shown in Fig.6.

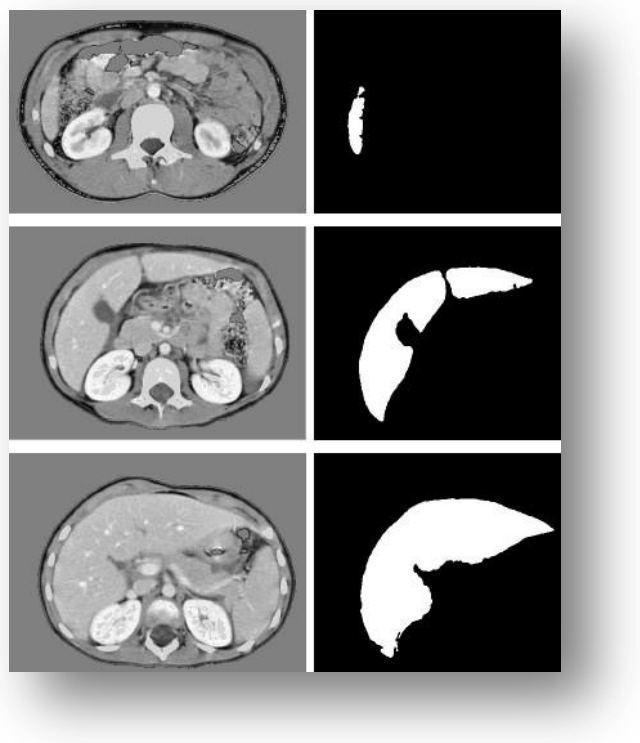

Fig. 6. Bayesian classifier segmentation results

Altarawneh et al. [30]in this paper researcher modify DLSR (Distance regularization level set) [1] method because it does not work well in case of weak or without edges of liver images. [30] overcome this issue by introducing new Balloon force that control weak and without the edges region by slowing down and controlling the evaluation process. Balloon force was created by utilizing probability density energy function to control the speed and energy of the evaluation process. Experiments were performed on 10 cases of $512 * 512$ pixels Slices. Graphical representation of comparison are shown in Fig. 7 below.

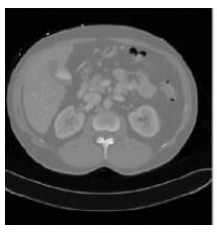

CT Slice

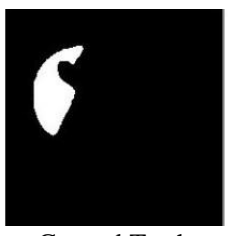

Ground Truth

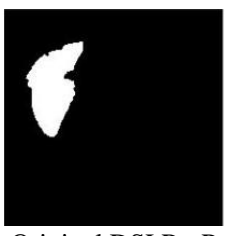

Original DSLR Proposed DSLR
Fig. 7. Comparison of DLSR with proposed DSLR with balloon force

\section{LIVER SEGMENTATION TECHNIQUES}

\section{A. Active contour}

Active Contour is the energy minimizing, deformable curves that moves due to the effects of internal and external object forces to detect object boundary. Internal forces prevent deformation. "The contours are classified broadly into two categories as: parametric active contours and geometric active contours. Parametric active contours are again classified into three types: Traditional snake, GVF snake and Ballon forces. Geometric active contours are Level set".

Basically, snakes use energy minimization to match a deformable model of an image.

General snake or active contour faces two problems:

- Initial model must be close to the boundary, otherwise it will show wrong result

- This model confronting issue to progress into boundary concavities.

AC [9][6][7] [13] model generally need manual initialization which is close to the image contour, otherwise it will give under or over segmentation problem. In case of liver segmentation, it causes over segmentation problem because liver share same intensity with the adjacent organs. It easily streams to the neighboring organ and cause over segmentation problem. Its performance is degraded if there should be an occurrence of irregular and higher intensities. Some of online available matlab codes are: [34] [35][36].

\section{B. Gradient vector flow}

Gradient Vector Flow [5] deal with two issues of the active contour model. It can converge into boundary concavities by calculating both concave and convex features. The traditional snake model must start close to the boundary while GVF [6][7] have the ability to start far from the boundary and till can converge to the image boundary. By and large, GVF demonstrate insensitivity to the initialization. GVF contour can handle broken object edges and subjective contour. Some of available code for GVF are in [37]

\section{Level set}

LS [13][16][30]semi-automatic techniques since it oblige user to select seed point.LS performance is very rely on the initial position, its performance increase when initial contour is placed close to the hepatic boundary .Its performance is degraded when the object is without edges or have weak edges[1]. The liver has the same intensity as the neighboring organs, hence cause over segmentation issues. Level set is time consuming for large computation. Level set strategy is additionally utilized for refinement of the liver segmentation. Some Matlab code accessible online is [38].

\section{Graph cut}

Graph Cuts, or max-flow/min-cut, is a generic technique for minimizing a specific form of energy called Markov random field (MRF) energy. In GC, image is represented using undirected weight graph. Each pixel represents every node of the graph. Each edge associated a couple of adjoining pixels. Similarity of grey level demonstrates the weight of edges between every match. Segmentation is the cut of graph. Every region speaks to a subgraph. The best cut is to make the subgraph similitude in a subgraph maximum and the closeness between the subgraph minimum. It is semi-automatic method since it requires user intervention for seed point selection which label the foreground and background. GC is not iterative method Graph cut is functioning admirably in homogenous area. Graph cut [3] can be made fully automatic 
using different algorithms. In case of liver tumor segmentation general active contour come up short when tumor is near liver surface, graph cut handle this kind of active contour issues extremely well.

\section{E. Adaptive thresholding}

Adaptive Thresholding [3] is additionally called local or dynamic thresholding. The principal idea of adaptive threshold is to apply different threshold on the different region of the image. Adaptive threshold divide the image into small areas and apply different threshold in different areas. On adaptive threshold value at each pixel location is depends on the neighboring pixel intensity. This type of thresholding is functioning admirably in irregular intensities and can handle the lightening condition extremely well. Adaptive threshold work on a pixel level, it sets all the pixel as foreground whose intensity value is greater than a certain threshold and all other pixels as background. Adaptive threshold work on color and grey scale image by converting it into a binary image. Adaptive threshold follow two approaches to find the threshold for every pixel: (i) the Chow and Kaneko approach and (ii) local thresholding. General online link for adaptive matlab code in [39].

\section{F. Region growing}

Region Growing [15][20][23][19]technique is semiautomatic strategy, since user interaction is required as the seed point is chosen by the user. RG method divides the image into regions according to predefine basis. This technique is initiates utilizing seed point, and examine the neighboring pixel either utilizing 4 connectivity or 8 connectivity, it iteratively adds the pixel to different region as indicated by predefined criteria. The criteria could be pixel intensity, gray level texture or color. In case of liver segmentation this technique gives great outcomes in contrast enhance images. Its effectiveness is relying on the determination of the seed point. Some of the RG code available online are [31][32][33].

\section{G. Fuzzy clustering mean(fcm)}

FCM is developed by dunn [40] is widely utilized in medical image processing. Its originate from the $\mathrm{k}$ mean algorithm. In $\mathrm{k}$ mean algorithm, each pixel is belonging to only one $\mathrm{k}$ cluster which is not feasible in case the of liver segmentation. FCM overcomes this issue by utilizing membership function which shows the belongings of the pixel to the cluster. FCM is fuzzy clustering technique which allows a pixel to belong to one or more clusters. FCM is a semiautomatic method. Some parameters like the selection of centroid, the degree of fuzziness and stopping criteria highly effects the performance of the FCM. In case of liver segmentation. FCM is mostly used for tumor segmentation [15] from the CT data.

\section{H. Statistical shape model(ssm)}

SSM [28][29][11] are find extremely effective in liver segmentation. The liver has an exceptionally varying shape. In this approach, probabilistic model is made to adapt to the varying shape of the liver. SSM can deal with the limitation of gray level techniques extremely well. During segmentation of liver, grey level techniques frequently appear under or over segmentation issues when the tumor is close to liver boundary.
This constraint of grey level techniques can handle utilizing SSM. Utilizing the prior knowledge SSM can deal unclear boundary of the liver extremely well. SSM focus on the shape of the liver. Limitation of shape based method and classifier based method [8][23]is the they require great number of training dataset.

\section{CONCLUSION}

The different segmentation techniques review has been done in this paper. Here the review of preprocessing, main segmentation technique, and their dataset information is provided in the form of a table. Comparative evaluation of different method is not possible in light of the fact that every author utilizes small private dataset set and different performance measure criteria are utilized. For objective comparison, for the most part acknowledge performance measures are required. From review of the techniques, it is concluded that there is still need to discover robust and efficient method for liver segmentation. As the liver is exceptionally difficult organ to handle and segment. Each existing method has its own advantage and disadvantages they are not full robust. Future work is to cover the classification techniques for liver tumor.

\section{REFERENCES}

[1] Li, Chunming, et al. "Distance regularized level set evolution and its application to image segmentation." IEEE transactions on image processing 19.12 (2010): 3243-3254.

[2] Lim, Seong-Jae, Yong-Yeon Jeong, and Yo-Sung Ho. "Automatic liver segmentation for volume measurement in CT Images." Journal of Visual Communication and Image Representation 17.4 (2006): 860-875.

[3] Massoptier, Laurent, and Sergio Casciaro. "Fully automatic liver segmentation through graph-cut technique." 2007 29th Annual International Conference of the IEEE Engineering in Medicine and Biology Society. IEEE, 2007.

[4] Rusko, Laszlo, et al. "Fully automatic liver segmentation for contrastenhanced CT images." MICCAI Wshp. 3D Segmentation in the Clinic: A Grand Challenge 2.7 (2007).

[5] Gui, Tianyi, Lin-Lin Huang, and Akinobu Shimizu. "Liver segmentation for CT images using an improved GGVF-snake." SICE, 2007 Annual Conference. IEEE, 2007.

[6] Alomari, Raja S., Suryaprakash Kompalli, and Vipin Chaudhary. "Segmentation of the liver from abdominal CT using Markov random field model and GVF snakes." Complex, Intelligent and Software Intensive Systems, 2008. CISIS 2008. International Conference on. IEEE, 2008.

[7] Massoptier, L., \& Casciaro, S. (2008). A new fully automatic and robust algorithm for fast segmentation of liver tissue and tumors from CT scans.European radiology, 18(8), 1658-1665.

[8] Mala, K., V. Sadasivam, and S. Alagappan. "Neural network based texture analysis of liver tumor from computed tomography images." International Journal of Biological and Life Sciences 2.1 (2006): 33-40.

[9] Jiang, Huiyan, and Qingshui Cheng. "Automatic 3D segmentation of CT images based on active contour models." Computer-Aided Design and Computer Graphics, 2009. CAD/Graphics' 09. 11th IEEE International Conference on. IEEE, 2009.

[10] Yussof, Wan Nural Jawahir Hj Wan, and Hans Burkhardt. "Integration of Morphology and Graph-based Techniques for Fully Automatic Liver Segmentation." Majlesi Journal of Electrical Engineering 4.3 (2010): 5966.

[11]Lamecker, Hans, Thomas Lange, and Martin Seebass. Segmentation of the liver using a 3D statistical shape model. Konrad-Zuse-Zentrum für Informationstechnik, 2004. 
[12] Militzer, Arne, et al. "Automatic detection and segmentation of focal liver lesions in contrast enhanced CT images." Pattern Recognition (ICPR), 2010 20th International Conference on. IEEE, 2010

[13] Suzuki, Kenji, et al. "Computer-aided measurement of liver volumes in CT by means of geodesic active contour segmentation coupled with level-set algorithms." Medical physics 37.5 (2010): 2159-2166.

[14] Wang, Ning, Lin-Lin Huang, and Baochang Zhang. "A fast hybrid method for interactive liver segmentation." Pattern Recognition (CCPR), 2010 Chinese Conference on. IEEE, 2010.

[15] Kumar, S. S., R. S. Moni, and J. Rajeesh. "Automatic segmentation of liver and tumor for CAD of liver." Journal of advances in information technology 2.1 (2011): 63-70.

[16] Li, Bing Nan, et al. "Integrating FCM and level sets for liver tumor segmentation." 13th International Conference on Biomedical Engineering. Springer Berlin Heidelberg, 2009.

[17] Belgherbi, A., I. Hadjidj, and A. Bessaid. "A Semi-automated Method for the Liver Lesion Extraction From a CT Images Based on Mathematical Morphology." Journal of Biomedical Sciences 2.2 (2013).

[18] Ciecholewski, Marcin. "Automatic liver segmentation from 2D CT images using an approximate contour model." Journal of Signal Processing Systems 74.2 (2014): 151-174.

[19] López-Mir, Fernando, et al. "Liver Segmentation on CT Images. A Fast Computational Method Based on 3D Morphology and a Statistical Filter." IWBBIO. 2013.

[20] Anter, Ahmed M., et al. "Automatic computer aided segmentation for liver and hepatic lesions using hybrid segmentations techniques." Computer Science and Information Systems (FedCSIS), 2013 Federated Conference on. IEEE, 2013.

[21] ALDEEK, NIDAA, et al. "LIVER SEGMENTATION FROM ABDOMEN CT IMAGES WITH BAYESIAN MODEL." Journal of Theoretical \& Applied Information Technology 60.3 (2014).

[22] Mostafa, Abdalla, et al. "CT liver segmentation using artificial bee colony optimisation." Procedia Computer Science 60 (2015): 1622-1630.

[23] Cheng, Ching-Hsue, and Liang-Ying Wei. "Rough Classifier Based on Region Growth Algorithm for Identifying Liver CT Image." 淡江理工學 刊 19.1 (2016): 65-74.

[24] Sayed, Gehad Ismail, Aboul Ella Hassanien, and Gerald Schaefer. "An Automated Computer-aided Diagnosis System for Abdominal CT Liver Images." Procedia Computer Science 90 (2016): 68-73.

[25] Sayed, Gehad Ismail, and Aboul Ella Hassanien. "Abdominal CT Liver Parenchyma Segmentation Based on Particle Swarm Optimization." The 1st International Conference on Advanced Intelligent System and Informatics (AISI2015), November 28-30, 2015, Beni Suef, Egypt. Springer International Publishing, 2016.

[26] Jemal, Ahmedin, et al. "Global cancer statistics." CA: a cancer journal for clinicians 61.2 (2011): 69-90.

[27] Wu, Kesheng, Ekow Otoo, and Arie Shoshani. "Optimizing connected component labeling algorithms." Medical Imaging. International Society for Optics and Photonics, 2005.

[28] Heimann, Tobias, H. Meinzer, and Ivo Wolf. "A Statistical Deformable Model for the Segmentation of Liver CT Volumes Using Extended Training Data." Proc. MICCAI Work (2007): 161-166.

[29] Li, Guodong, et al. "Automatic liver segmentation based on shape constraints and deformable graph cut in CT images." IEEE Transactions on Image Processing 24.12 (2015): 5315-5329.

[30] Altarawneh, Nuseiba, et al. "Liver Segmentation from CT Images Using a Modified Distance Regularized Level Set Model Based on a Novel Balloon Force| NOVA. The University of Newcastle's Digital Repository." (2014).

[31] https://www.mathworks.com/matlabcentral/fileexchange/50782-regiongrowing-segmentation

[32] https://www.mathworks.com/matlabcentral/fileexchange/32532-regiongrowing--2d-3d-grayscale-

[33] https://www.mathworks.com/matlabcentral/fileexchange/19084-regiongrowing

[34] https://www.mathworks.com/matlabcentral/fileexchange/19567-activecontour-segmentation

[35] https://www.mathworks.com/matlabcentral/fileexchange/23445-chanvese-active-contours-without-edges

[36] http://www.shawnlankton.com/2008/04/active-contour-matlab-codedemo/

[37] https://www.mathworks.com/matlabcentral/fileexchange/45896-fastgradient-vector-flow--gvf-

[38] https://www.mathworks.com/matlabcentral/fileexchange/12711-level-setfor-image-segmentation

[39] https://www.mathworks.com/matlabcentral/fileexchange/8647-localadaptive-thresholding

[40] Dunn, Joseph C. "A fuzzy relative of the ISODATA process and its use in detecting compact well-separated clusters." (1973): 32-57. 


\section{TABLE I. COMPARISION OF DATASET}

\begin{tabular}{|c|c|c|c|c|c|}
\hline Ref & Author & Year & Images used & Datatypes & Image size \\
\hline [2] & Lim et al & 2005 & 10cases & Private dataset & $512 * 512$ \\
\hline [3] & Massoptier et al & 2007 & 10cases & Private dataset & - \\
\hline [4] & Rusko et al & 2007 & 10cases & - & - \\
\hline [5] & Gui et al & 2007 & 4cases ,200images & Private dataset & $170 * 170$ \\
\hline [6] & Alomari et al & 2008 & 13 cases & Private dataset & $512 * 512$ \\
\hline [7] & Massoptier et al & 2008 & 21cases & Private dataset & $512 * 512$ \\
\hline [8] & Mala et al & 2008 & 105 images & Private dataset & $256 * 256$ \\
\hline [9] & Jiang et al & 2009 & 5 cases & - & - \\
\hline [10] & Yussof et al & 2010 & 10cases & - & $512 * 512$ \\
\hline [11] & Akram et al & 2010 & 100images & Private dataset & $512 * 512$ \\
\hline [12] & Militzer et al & 2010 & 15 cases & Private dataset & - \\
\hline [13] & Suzuki et al & 2010 & 15cases & Private dataset & - \\
\hline [14] & Wang et al & 2010 & 100images & Private dataset & $512 * 512$ \\
\hline [15] & S.S Kumar & 2011 & 10cases & - & $512 * 512$ \\
\hline [16] & Li et al & 2009 & 15 cases & $\begin{array}{l}\text { LTSCdataset } \\
\text { NUHdataset }\end{array}$ & $512 * 512$ \\
\hline [17] & Belgerbi et al & 2013 & - & Private dataset & - \\
\hline [18] & Ciecholewski & 2014 & $\begin{array}{l}1330 \text { images } \\
120 \text { cases } \\
\end{array}$ & Private dataset & - \\
\hline [19] & Lopez-mir et al & 2013 & 30 cases & Private dataset & $512 * 512$ \\
\hline [20] & M.Anther et al & 2013 & $\begin{array}{l}112 \text { cases } \\
860 \text { images }\end{array}$ & 2 Private datasets & $630 * 630$ \\
\hline [21] & ALDEEK et al & 2014 & 44cases & Private dataset & $512 * 512$ \\
\hline [22] & Mostafa, et al & 2015 & 38CT images & Private dataset & - \\
\hline [23] & Cheng et al & 2016 & 800images & $\begin{array}{l}\text { Open NBIA } \\
\text { Private dataset }\end{array}$ & $512 * 512$ \\
\hline [24] & Sayed et al & 2016 & 62 Images & - & $256 * 256$ \\
\hline [25] & Sayed et al & 2016 & 43 images & - & $256 * 256$ \\
\hline
\end{tabular}


TABLE II. LIVER SEgMENTATION TECHNIQUES OVERVIEW

\begin{tabular}{|c|c|c|c|}
\hline REF NO & PRE-PROCESSING & LIVER SEGMENTATION & EVALUATION \\
\hline [3] & $\begin{array}{l}\text { Mean shift filter } \\
\text { Adaptive threshold }\end{array}$ & Graph cut & Accuracy $96 \%$ \\
\hline [4] & $\begin{array}{l}\text { Hough transform } \\
\text { Erosion/dilation } \\
\text { Largest CCL }\end{array}$ & Region growing & Accuracy $76 \%$ \\
\hline [5] & $\begin{array}{l}\text { Canny edge detector } \\
\text { Hermit spline curve } \\
\text { Anisotropic diffusion filter }\end{array}$ & GGVF & - \\
\hline [2] & $\begin{array}{l}\text { Multilevel thresholding } \\
\text { Morphological filter }\end{array}$ & $\begin{array}{l}\text { Gradient label map } \\
\text { K mean clustering } \\
\text { Label based search algorithm }\end{array}$ & Accuracy $96 \%$ \\
\hline [6] & $\begin{array}{l}\text { Histogram analysis } \\
\text { Markov random field }\end{array}$ & $\begin{array}{l}\text { Gradient vector flow } \\
\text { Active contour }\end{array}$ & Similarity matrix \\
\hline [7] & $\begin{array}{l}\text { Adaptive threshold } \\
\text { Mean shift filter }\end{array}$ & $\begin{array}{l}\text { Gradient vector flow } \\
\text { Active contour }\end{array}$ & $\begin{array}{l}\text { A volume overlap of liver } 94.2 \% \\
\text { Sensitivity \&specificity for tumor } \\
82.6 \% \& 87.5 \% \text { respectively }\end{array}$ \\
\hline [9] & $\begin{array}{l}\text { Sobel operator } \\
\text { Erosion/dilation }\end{array}$ & Active contour & Accuracy $94 \%$ \\
\hline [10] & $\begin{array}{l}\text { Opening } \\
\text { Multiscale filtering } \\
\text { Anisotropic diffusion filter }\end{array}$ & $\begin{array}{l}\text { CCL } \\
\text { Graph Cut }\end{array}$ & - \\
\hline [11] & $\begin{array}{l}\text { Median filter } \\
\text { Adaptive Histogram } \\
\text { Power law transformation }\end{array}$ & $\begin{array}{l}\text { Closing } \\
\text { Largest area } \\
\text { Global threshold }\end{array}$ & Accuracy $96 \%$ \\
\hline [13] & $\begin{array}{l}\text { Anisotropic diffusion filter } \\
\text { Median filter } \\
\text { Scale specific gradient magnitude filter }\end{array}$ & $\begin{array}{l}\text { Fast marching } \\
\text { Level set } \\
\text { Geodesic active contour }\end{array}$ & Manual tracing method \\
\hline [14] & $\begin{array}{l}\text { Gradient magnitude } \\
\text { Anisotropic diffusion filter }\end{array}$ & $\begin{array}{l}\text { Random walk algorithm } \\
\text { Watershed }\end{array}$ & $\begin{array}{l}\text { TP, TN, FP, FN } \\
\text { Manual tracing method }\end{array}$ \\
\hline [15] & $\begin{array}{l}\text { Median filter/ Erosion } \\
\text { Largest CCL }\end{array}$ & $\begin{array}{l}\text { Region growing } \\
\text { Alternate FCM }\end{array}$ & Manual tracing method \\
\hline [16] & Morphological operations & $\begin{array}{l}\text { Fuzzy Clustering Mean/ Level set } \\
\text { Balloon force }\end{array}$ & - \\
\hline [17] & $\begin{array}{l}\text { LIVER: } \\
\text { H max transform } \\
\text { Dilation/erosion } \\
\text { Lesion: } \\
\text { Anisotropic diffusion filter } \\
\text { Hmaxima transform filter }\end{array}$ & Watershed algorithm & $\begin{array}{l}\text { Sensitivity \& specificity } \\
92 \% \text { \& } 99 \% \text { respectively }\end{array}$ \\
\hline [18] & - & Connected poly lines & DICE similarity co efficient $81.3 \%$ \\
\hline [19] & $\begin{array}{l}\text { Adaptive filter } \\
\text { Dilation/erosion }\end{array}$ & Region Growing & - \\
\hline [20] & $\begin{array}{l}\text { Erosion/dilation } \\
\text { Adaptive threshold }\end{array}$ & $\begin{array}{l}\text { CCL } \\
\text { Region Growing } \\
\text { Watershed }\end{array}$ & Accuracy $96 \%$ \\
\hline [21] & $\begin{array}{l}\text { Convert intensity value into Hounsfield units } \\
\text { Median filter }\end{array}$ & Bayesian Model & Accuracy $87 \%$ \\
\hline [22] & $\begin{array}{l}\text { Median filter } \\
\text { Contrast Stretching } \\
\text { Thresholding to separate Ribs }\end{array}$ & $\begin{array}{l}\text { Artificial bee colony } \\
\text { Optimization algorithm }\end{array}$ & Accuracy $93.73 \%$ \\
\hline [24] & Median filter & $\begin{array}{l}\text { FCM } \\
\text { Grey Wolf Optimization } \\
\text { SVM }\end{array}$ & Accuracy $96 \%$ \\
\hline
\end{tabular}

